\title{
LINKING BUSINESS ANALYTICS TO DECISION MAKING EFFECTIVENESS: A PATH MODEL ANALYSIS
}

Key words: Business analytics; Information processing capability; Decision-making effectiveness; Information processing view; Contingency theory; Data-driven environment

\begin{abstract}
While business analytics is being increasingly used to gain data-driven insights to support decision-making, little research exists regarding the mechanism through which business analytics can be used to improve decision-making effectiveness at the organisational level. Drawing on the information processing view and contingency theory, this paper develops a research model linking business analytics to organisational decision-making effectiveness. The research model is tested using structural equation modelling based on 740 responses collected from UK businesses. The key findings demonstrate that business analytics, through the mediation of a data-driven environment, positively influences information processing capabilities, which in turn have a positive effect on decision-making effectiveness. The findings also demonstrate that the paths from business analytics to decision-making effectiveness have no statistical differences between large and medium companies but some differences between manufacturing and professional service industries. Our findings contribute to the business analytics literature by providing useful insights into business analytics applications and the facilitation of data-driven decision-making. They also contribute to managers' knowledge and understanding by demonstrating how business analytics should be implemented to improve decision-making effectiveness.
\end{abstract}




\section{INTRODUCTION}

Business analytics (BA) refers to "the extensive use of data, statistical and quantitative analysis, explanatory and predictive models, and fact-based management to drive decisions and actions" [1, pp. 7]. The concept of BA was initially developed in the mid-1950s and has been widely examined over the years $[2,3]$. However, BA has recently re-emerged as an important area of study [3-5]. Several key reasons can be identified for the growing importance of BA. First, the advances in information technology (IT) have enabled businesses to develop innovative ways to collect data from both internal and external sources [2]. This leads to the unprecedented challenges of big data, characterised by "high volume, high velocity, and/or high variety" [4, pp. 1249], as processing big data is difficult and requires new and advanced technologies [3]. At the same time, big data offers remarkable business opportunities for organisations to gain useful insights into customers and operations [4]. Consequently, BA, based on sophisticated IT [6, 7], has been increasingly used by organisations [4, 7-9]. Second, organisations require BA to "gain an edge by making better or faster decisions" [10, pp. 30] to face increasing competition and turbulence in their marketplaces due to the speed of technological advancement and globalisation. Third and most importantly, the confluence of big data, advances in IT, and BA, has brought decisionmaking to a completely new level that is ever so data-driven, allowing managers to see what was previously invisible [11]. This represents "a qualitative change in opportunities to generate value and competitive advantage", and to enable decision-making move towards "territory which has historically been seen as reliant on human judgment" [12, pp. 288-289].

Despite the importance of BA and data-driven decision-making [7-9], surprisingly little academic research has been conducted to understand BA as an emerging field of study $[5,13]$. Consequently, little is known about the mechanisms through which BA improves decisionmaking. As many companies are still struggling to figure out how to use analytics $[8,11,14]$, 
the absence of such an understanding limits the ability of businesses to effectively leverage BA for value creation. Until the mechanisms through which BA influences organisational decision-making is better understood, realising business value from BA remains a challenge.

This paper therefore aims to reduce this research gap by developing an understanding of the mechanisms through which BA improves decision-making effectiveness that is the extent to which a decision results in desired outcomes [15]. Drawing on the information processing view and contingency theory, this paper develops and empirically tests a path model to explain how BA and other organisational factors work together to enhance decisionmaking effectiveness.

Although contingency theory and the information processing view have been used previously to understand the impact of IT on organisations, no research based on these two theories has been conducted to date to examine the emerging BA and its impact on decisionmaking effectiveness. Thus, this research seeks to contribute to the literature by developing a research model in which relevant constructs regarding BA's impact on decision-making effectiveness are conceptualised and tested. To evaluate this research model empirically, partial least squares structural equation modelling (PLS-SEM) is used, based on 740 responses that are collected from an online questionnaire survey of UK businesses. A multigroup analysis is also conducted to understand whether industry and firm size moderate the relationships hypothesised in the research model. This study shows that BA supported with a data-driven environment will lead to the development of information processing capabilities, which in turn have a major impact on organisational decision-making and decision-making effectiveness. This research will also contribute to managers' knowledge and understanding of BA and its impact thereby to improve organisational decision-making.

The next section of the paper presents the literature review, the research model, and hypotheses. The subsequent sections describe the instrument development and the data 
collection processes, and report on the findings. The final section discusses the results and implications.

\section{THEORETICAL BACKGROUND}

This section begins with defining the key terms to be used in this paper and then develops hypotheses regarding the effect of BA on decision-making effectiveness.

\subsection{Key concepts defined}

From the information processing view $[16,17]$, the key task for organisations is to manage uncertainty such as task complexity and the rate of environmental change through deploying mechanisms of information processing. The information processing view emphasises the importance of matching information processing requirements with information processing capabilities: the greater the task uncertainty, the greater amount of information that has to be processed [16]. Therefore, organisations should design its structure [17] or business processes [18] to facilitate information processing to enable decision makers to process a great amount of data, thereby to inform decision-making, reduce costs, and improve organisational performance. For instance, [18] demonstrates that the interactive effect of information processing needs and information processing capabilities has a significant effect on performance in an inter-organisational supply chain context. Likewise, [19] shows that there is a positive relationship between inter-firm information processing capabilities and supply chain company performances. Thus, an organisation is expected to be more effective when its information processing requirements are matched by its information processing capabilities [17].

The concept of information processing capabilities is initially used by [16] without a definition to outline the information processing view of organisational design. These terms are adopted by [17, pp. 614] to further develop the information processing view, while information processing is defined as "the gathering, interpreting, and synthesis of 
information in the context of organizational decision making". Based on the information processing view and BA studies [7-9, 20], information processing capabilities of an organisation can be defined as its capacities to capture, integrate and analyse data and information, and use the insights gained from data and information in the context of organisational decision-making.

The next key concept to be discussed is an organisation's data-driven environment that is the organisational practices reflected by developing explicit strategy and policy to guide analytic activities and designing its structure and processes to enable and facilitate BA activities. [21, pp. 22] suggests that "for analytics-driven insights to be consumed - that is, to trigger new actions across the organization--they must be closely linked to business strategy, easy for end-users to understand and embedded into organizational processes so action can be taken at the right time". Similarly, it is argued that it is vital to develop an "analytically driven strategy" [1], relevant business processes [11], and organisational structure [22] so that BA can be embedded into organisational practices thereby to improve decision-making and decision-making effectiveness. Otherwise, "a company will not know on which data to focus, how to allocate analytic resources, or what it is trying to accomplish in a data-toknowledge initiative" [7, pp. 122]. Thus, in order for an organisation to use BA effectively to create business value, a data-driven environment must be created by developing specific organisational strategy, policy, structure, and business processes to support and enable BA activities $[7-9,20]$.

Accordingly, data-driven decision-making can be defined as the extent to which an organisation is open to new ideas that challenge current practice based on data-driven insight; has the data to make decisions; and depends on data-based insights for decision-making and the creation of new service or product $[8,9,20]$. Hence, decision-making effectiveness can be 
specified as the extent to which a company is more effective than its competitors at making real-time decisions, responding to change, and understanding customers, based on $[15,23]$.

\subsection{BA and information processing capabilities}

Prior BA studies [e.g., 7, 8, 9, 20] suggest that the application of BA in an organisation is likely to enhance the organisation's abilities to process data and to use the insights derived from that data to make effective decisions, thereby to improve organisational performance. Thus, based on the definition of BA and that of information processing capabilities, we propose:

H1: BA has a positive and direct effect on information processing capabilities.

However, the causal link from BA to information processing capabilities is much more complex than this direct relationship could describe. Prior BA studies have indicated that in order for a business to benefit from BA, simultaneously the business needs to develop a datadriven environment to support BA applications [4, 7-9, 20]. Essentially this suggests a degree of fit between BA and a data-driven environment, and the nature and the importance of this fit can be better understood drawing on contingency theory.

Contingency theory defines fit as "the degree to which the needs, demands, goals, objectives, and/or structures of one component are consistent with the needs, demands, goals, objectives, and/or structures of another component" [24, pp. 45], and conjectures that performance is a consequence of that fit [25]. Contingency theory has been extensively applied to examining the relationships between, for example, IT, organisational factors, and organisational performance [e.g., 26, 27]. These IT business value studies suggest that when IT and organisational factors are integrated, together they are seen to be able to generate various types of IT capabilities [e.g., 28, 29], which in turn enable an organisation to leverage technology to differentiate from competition [30]. Inspired by IT business value studies and drawing on extant BA studies, the fit between BA and a data-driven environment in an 
organisation can be argued to have a positive impact on the organisation's information processing capabilities. It can be expected that an organisation with a higher degree of fit between its BA and data-driven environment will outperform those with lower degree of fit; and the better the fit, the stronger the information processing capabilities.

Regarding how this fit influences information processing capabilities, a mediation model of fit can be supported by the proposition that technology can be an important determinant of organisational processes and structure in research underpinned by contingency theory [31]. For example, [32] argues that increasing technological complexity would require greater structural complexity for effective performance, while [33] suggests that technology can be a determinant of organisational processes and structure. Alternatively, [34] examines the relative routineness of work and advocated that organisational structure depends on technology. In line with this, it can be argued that BA applications are likely to bring about a data-driven environment embedded in and reflected by explicitly developing organisational strategy, policy, structure, and business processes to guide and enable BA activities, which will help develop information processing capabilities. Thus, it is proposed that

H2: BA has a positive and indirect effect on information processing capabilities through the mediation of a data-driven environment.

\subsection{Data-driven environment, information processing capabilities and decision- making}

Drawing on the information processing view, an organisation is more likely to make effective decisions when it designs its structure [17] and business processes [18] to facilitate its information processing capabilities thereby to meet its data processing requirements. For instance, the processing requirement of big data is complex as it involves dealing with data that are high in volume, variety, and velocity. This big data processing requirement is overwhelming to organisations since "it is very difficult for individuals to process large 
volumes of incoming information comprehensively" [35, pp. 156]. It is also impossible for traditional systems to capture, store, and analyse big data $[2,7]$; rather, it requires new and innovative forms of information processing capabilities that are likely to be provided by BA with "advanced and unique data storage, management, analysis, and visualization technologies" [3, pp. 1166]. Therefore, in order for an organisation to meet its big data processing needs, it must develop its information processing capabilities through effective BA applications, which are enabled by developing an "analytically driven strategy" [1] and designing relevant business processes [11] and organisational structure [22].

When an organisation has developed strong information processing capabilities to match its data processing requirements, the organisation can be expected to have sufficient information and data-driven insights to allow it to evaluate its business practices, to make informed decisions not only to improve internal business efficiencies but also to create new products or services for customers [2], to achieve faster cycle times and greater flexibility [6], and/or to significantly improve its performance [16]. This is consistent with the strategic decision-making research. For example, it is expected that when a business has complete and accurate information about the relationship between choices and outcomes, it will be most likely to make successful decisions [36], to generate viable organisational strategies [37], and to improve organisational performance [38]. Therefore, it is proposed that

H3: Information processing capabilities have a positive effect on a data-driven decision-making.

H4: Information processing capabilities have a positive and direct effect on decisionmaking effectiveness.

Furthermore, it has been widely recognised in the BA literature that the potentials of BA can only be realised when a data-driven environment is developed so that decisionmaking, strategy, and operations rely on data-driven insights $[1,8,9]$. A data-driven 
environment is seen to help a company to have the data to make decisions, to be open to new ideas, to make decisions depending on fact-based insights, and to use fact-based insight for the creation of new service or product. Thus, it is proposed that

H5: A data-driven environment is positively and directly associated with data-driven decision-making.

H6: Data-driven decision-making is positively associated with decision-making effectiveness.

\subsection{The moderating effect of firm size and industry type}

The relationship between IT and firm size is an important area of study [27, 39]. Firm size matters because it may affect the relationship between IT and other organisational aspects such as the use and spending patterns of IT investment [40, 41]. This paper is particularly interested in whether firm size might affect the way organisations implement BA.

Prior research has reported in the IT context that firm size has a moderating effect on for example the total effects of quality system on final outcome [42] or weakly on the performance relationship of advanced manufacturing technology [43]. In other areas of management research, the findings on the moderating impact of firm size are at variance [e.g., $44,45]$. Nevertheless, the impact of firm size should not be ignored. This research examines whether firm size moderates the paths from BA to decision-making effectiveness. As prior studies indicate that companies with different sizes behave differently regarding IT use and investment $[40,41]$, it is thus proposed that

H7: Firm size moderates the paths from BA to decision-making effectiveness.

Another important variable is industry type since firms in different industries often differ systematically regarding IT spending, needs for IT, and other organisational and technological conditions that are relevant to the way IT is used [46]. While the impact of industry type on IT has received limited attention in IT research [46], prior studies in other 
research areas, however, have found support for the moderating effect of industry type on organisational performance [e.g., 47, 48]. Similarly, it is expected that industry type is likely to play a moderating role in affecting BA applications. Thus, it is proposed that

H8: Industry type moderates the paths from BA to decision-making effectiveness. As a result, our research model can be summarised in Figure 1.

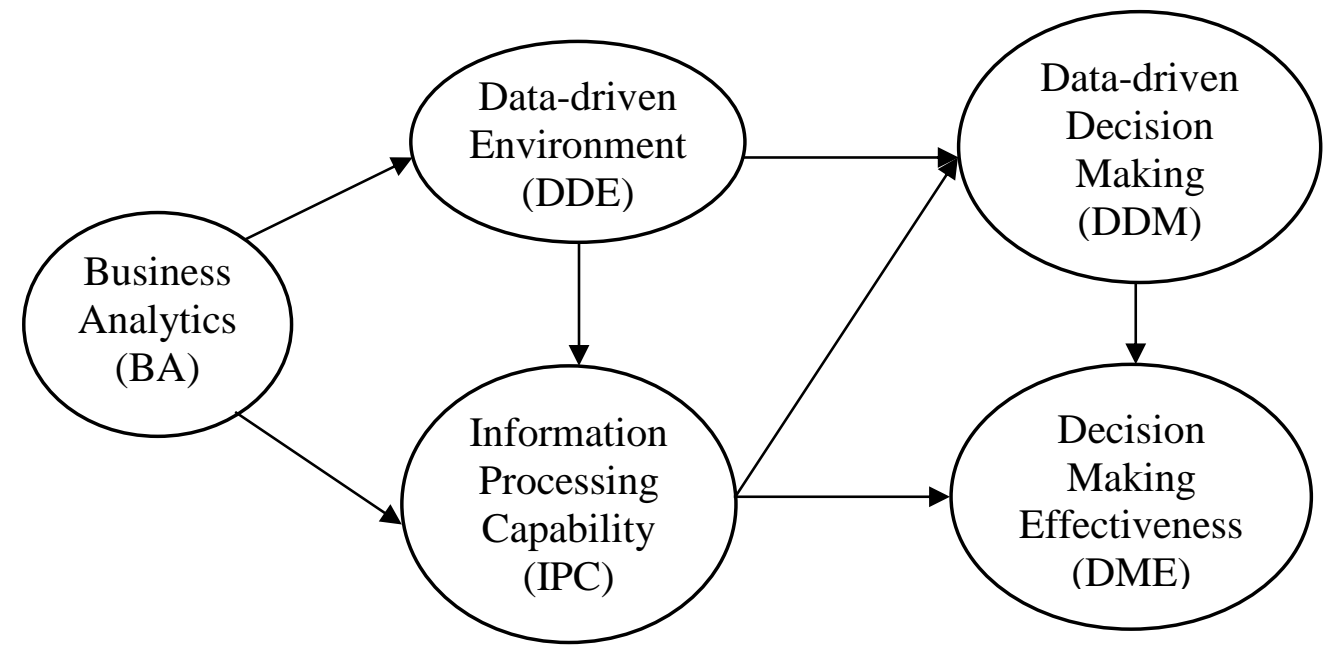

Figure 1. Research model

\section{RESEARCH METHOD}

The hypotheses are tested based on survey data using PLS-SEM. PLS-SEM is recommended to be well suited for research situations where theory is less developed [49-51] and the objective is prediction or to explain relationships among a set of constructs in research situations where the phenomenon under study is new [52-54]. The importance of BA may have been widely discussed, but BA is still re-emerging as a new research area while extant BA studies are "predominantly practice driven ...there is very little published management scholarship" [13, pp. 321]. Consequently, there are hardly any developed measures for new constructs in this area and few empirical studies to shed light on the relationships between BA and other organisational variables. Thus, PLS-SEM is considered appropriate for the present study to conceptualise and empirically test the paths from BA to decision-making effectiveness. PLS-SEM is also appropriate for the present study as it can handle both 
reflective and formative constructs, both of which are used in the research model. In the following section, we outline the instrument development, validation, and dissemination processes.

\subsection{Research model constructs}

To develop and test the research model, a number of constructs are identified and are summarised in Table 1. As BA is still re-emerging as a new research area, there are few previously validated measurement items. Thus, five new formative constructs have been developed for this research based on literature on BA and IT business value.

To properly develop formative constructs is challenging [54] as the scale development procedures suggested in the literature are limited [55]. Failing to define constructs properly may cause serious problems such as damaging the validity of the constructs and statistical conclusions [55] and/or affecting theory development and theory testing [56]. In order to avoid common misspecifications, we develop the five constructs based on the four decision rules [56]: the direction of causality between construct and indicators, the interchangeability of indicators, the covariation among indicators, and the nomological net for the indicators. To make the development process more transparent and robust, the definition of BA is used as an example. Based on prior research [e.g., 3, 7, 9], BA is defined formatively by 13 different indicators in two stages: before and after data collection [56]. Prior to data collection, the first decision rule considered is the direction of causality between BA and its indicators. Rather than BA defines the indicators, it is more appropriate to understand BA as a composite concept formed jointly by its indicators, each of which clearly captures different aspects of the construct. For example, while web analytics focuses on digital data analysis, simulation and model management are different and mainly about modelling. Thus, changes in each indicator would have caused change in how BA is defined and interpreted. Second, are the indicators interchangeable? Web analytics and social media analytics for instance 
Table 1. Constructs and indicators of the study

\begin{tabular}{|c|c|c|}
\hline Constructs & Indicators & Reference \\
\hline BA & $\begin{array}{l}\text { How often does your organisation use the following? } \\
\text { Statistical analysis (SA1) } \\
\text { Forecasting (FC1) } \\
\text { Query and analysis (QA1) } \\
\text { Predictive modelling (PM1) } \\
\text { Optimisation (OPT1) } \\
\text { Model management (MM1) } \\
\text { Simulation \& scenario development (SM1) } \\
\text { Business reporting /KPIs/Dashboards (KPI1) } \\
\text { Web analytics (WA1) } \\
\text { Social media analytics (SMA1) } \\
\text { Interactive data visualisation (IDV1) } \\
\text { Text, audio, video analytics (TAVA1) } \\
\text { Data and text mining (DTM1) }\end{array}$ & {$[3,7,9]$} \\
\hline $\begin{array}{l}\text { Data-Driven } \\
\text { Environment } \\
\text { (DDE) }\end{array}$ & $\begin{array}{l}\text { To what extent do you agree or disagree } \\
\text { We have explicit organisational strategy that guides business } \\
\text { analytics activities (STRA1) } \\
\text { We have explicit policies and rules that guide business } \\
\text { analytics activities (POL1) } \\
\text { We have well-defined organisational structure that enables } \\
\text { business analytics activities (STRU1) } \\
\text { Business analytics is integrated into our business processes } \\
\text { (PRO1) } \\
\text { We prioritise major business analytics investments by the } \\
\text { expected impact on business performance (PERF1) }\end{array}$ & $\begin{array}{l}{[2,7,8,} \\
20]\end{array}$ \\
\hline $\begin{array}{l}\text { Information } \\
\text { processing } \\
\text { capabilities } \\
\text { (IPC) }\end{array}$ & $\begin{array}{l}\text { We are more effective than our competitors at } \\
\text { Capturing data/information (CD1) } \\
\text { Integrating data/information (ID1) } \\
\text { Analysing data/information (AD1) } \\
\text { Using insights gained from data/information (UD1) } \\
\end{array}$ & {$[7-9,20]$} \\
\hline $\begin{array}{l}\text { Data-driven } \\
\text { Decision } \\
\text { Making } \\
(\mathrm{DDM})\end{array}$ & $\begin{array}{l}\text { To what extent do you agree or disagree } \\
\text { We use data-based insight for the creation of new } \\
\text { service/product (S/P1) } \\
\text { We depend on data-based insights for decision making } \\
\text { (DM1) } \\
\text { We are open to new ideas that challenge current practice } \\
\text { based on data-driven insight (OPEN1) } \\
\text { We have the data to make decisions (DATA1) }\end{array}$ & {$[2,8,20]$} \\
\hline $\begin{array}{l}\text { Decision } \\
\text { Making } \\
\text { Effectiveness } \\
\text { (DME) }\end{array}$ & $\begin{array}{l}\text { We are more effective than our competitors at } \\
\text { Responding quickly to change (CHA1) } \\
\text { Making real-time decisions (RTD1) } \\
\text { Understanding customers (CUS1) }\end{array}$ & $\begin{array}{l}{[8,9,} \\
20]\end{array}$ \\
\hline
\end{tabular}

share a common theme focusing on digital data analysis, but they are distinctly different from optimisation and model management that focus on modelling. Thus, the indicators are not 
interchangeable and the elimination of indicators may affect the characteristics of BA. Third, are the indicators expected to covary with each other? The answer to this is not simply positive or negative. It could be expected that indicators focusing on the same theme such as analysing digital data are more likely to covary than those having different themes are. Thus BA seems to be multidimensional than unidimensional, which could be verified by conducting a factor analysis after data collection. Finally, regarding whether the indicators have the same antecedents and consequences, the answer is not necessary. For example using web analytics to analyse digital data may be driven by e-commerce initiatives while modelling can be enacted by any business practices; accordingly, their consequences may differ. This consideration again suggests that BA should be defined as a multidimensional construct. For example, indicators focusing on digital data analysis should be grouped together and defined as a reflective construct because they share a common theme and tend to be interchangeable; the same should also be applicable to indicators relating to modelling. Thus, prior to data collection, it is seen to be more appropriate to define BA formatively as a higher-order component by a few lower-order reflective components. The reflective lowerorder components are then determined based on an exploratory factor analysis after data are collected, which is covered in Section 4.4. Similarly, other formative constructs are defined based on the four decision rules.

\subsection{Data collection}

To test the hypotheses empirically, we have selected both medium (with employees between 50 and 250) and large (more than 250 employees) UK enterprises as they are expected to have the expertise and resources to employ various types of BA. A questionnaire survey is generated using a five-point Likert scale measurements for all constructs. The survey instruments are developed based on literature review initially and then are scrutinised by five internal subject experts. After a few revisions, the survey is piloted to ensure that the 
respondents understand the questions and there are no problems with the wording or measurements. The survey is then delivered electronically through Qualtrics to managers, whose email addresses are identified from the FAME database. Three rounds, four weeks apart, of emails including the survey are sent. Each intended respondent is entered into a draw to win an iPad mini and is offered a summary of the results. While 103,000 emails are sent with the e-mail subject highlighted as questionnaire survey, the majority of them are never opened; though a few companies have replied to state that they have a policy not to participate in any surveys. Of all sent emails, 2,276 are opened, representing a click-through rate of $2.2 \%$; of these opened, we have received 740 usable responses, which represent a $32.5 \%$ response rate.

\section{$4 \quad$ RESULTS}

\subsection{Respondent's profile}

Table 2 summarises the respondents' characteristics in terms of their organisational positions and years of experience in their current firms and industry.

Table 2. Respondent profiles $(n=740)$

\begin{tabular}{|l|c|l|c|}
\hline Industry & $\%$ & Positions & $\%$ \\
\hline Manufacturing & 31 & CEO/MD/Partner & 28 \\
\hline Prof Services & 15 & Finance/Accounting director & 13 \\
\hline Retail/Wholesale & 8 & Operations director & 11 \\
\hline Technology & 7 & Marketing/Sales director & 11 \\
\hline Financial Services & 6 & CIO/IT Manager & 8 \\
\hline Other & 33 & Other directors & 29 \\
\hline
\end{tabular}

\begin{tabular}{|l|c|c|}
\hline \multicolumn{3}{|c|}{ Respondent Experience } \\
\hline Years & In the firm \% & In the industry \% \\
\hline$\leq 5$ & 22 & 4 \\
\hline $5<$ but $\leq 10$ & 29 & 10 \\
\hline $10<$ but $\leq 15$ & 13 & 12 \\
\hline $15<$ but $\leq 20$ & 12 & 15 \\
\hline $20<$ but $\leq 25$ & 10 & 14 \\
\hline$>25$ & 14 & 45 \\
\hline
\end{tabular}


A key informant approach is used to collect data [57]. The reported positions of the respondents suggest that $28 \%$ of the respondents are in a senior managerial position and the rest of them are middle managers. Based on their managerial positions, the respondents are highly likely to participate in decision-making processes related to the topic of the survey [58]. Of all respondents, $49 \%$ have been with their firms for more than 10 years, whilst $86 \%$ have been in the industry for more than 10 years. The respondents are from a number of different industries, for example $31 \%$ from manufacturing sector, $15 \%$ from professional services, and $8 \%$ from retail/wholesale. Overall, the sample of respondents seems to be diverse, representing various industry, managerial position and experience.

\subsection{Common method and non-respondent bias}

Common method bias that may affect the correlations between variables and cause biased parameter estimates [59] is assessed by conducting an exploratory factor analysis (EFA). Harman's single-factor test is conducted by entering all independent and dependent variables [60]. If a single factor explains most of the variance of all the indicators, then the common method variance (CMV) associated with the data is high. Conversely, if more than one factor emerges to explain most of the communality, then the CMV associated with the data is low. In this research, the test result shows that the first factor accounts for $33.22 \%$ of the total variance; there is no evidence of a substantial amount of CMV in the data.

To evaluate the presence of non-response bias, we conduct two tests. The first test compares the distributions of the position and company size of the respondents with those of the complete sampling frame (respondents plus non-respondents with e-mail addresses), based on the known value for the population approach [61]. In Table 3, the position and company size of the respondents are the observed values, while the position and company size of the members of the full sampling frame are the expected values. If the observed and the expected values are significantly different, there is a bias between respondents and non- 
respondents. A nonparametric chi-square test comparing the distributions of the observed and expected values finds no significant differences.

Table 3. Expected and observed value

\begin{tabular}{|c|c|c|}
\hline Position & Observed value (\%) & Expected value (\%) \\
\hline CEO/MD/Partner & 28 & 10 \\
\hline Finance/Accounting director & 13 & 7 \\
\hline Operations director & 11 & 2 \\
\hline Marketing/Sales director & 11 & 8 \\
\hline CIO/IT Manager & 8 & 6 \\
\hline \multicolumn{3}{|c|}{ Chi-square test $p$-value $=0.9387$} \\
\hline Company size & Observed value (\%) & Expected value (\%) \\
\hline Medium & 71 & 67 \\
\hline Large & 29 & 33 \\
\hline \multicolumn{3}{|c|}{ Chi-square test $p$-value $=0.9322$} \\
\hline
\end{tabular}

As a second test for non-response bias, we compares early $(n=350)$ and late $(n=390)$ respondents, based on the premise that early respondents represent the average respondent while late respondents represent the average non-respondent [61]. All 29 indicators are evaluated by comparing the two groups through an independent $t$-test. The t-test results yield two statistically significant differences: MM1 (one of 13 BA indicators) scores are significant at the $\mathrm{p}=0.008<0.05$ (two-tailed) for early respondents $(\mathrm{M}=2.429, \mathrm{SD}=1.2253)$ and late respondents $(\mathrm{M}=2.160, \mathrm{SD}=1.0952)$; and $\mathrm{OPEN1}$ (one of four data-driven decision-making indicators) scores are significant at the $\mathrm{p}=0.033<0.05$ (two-tailed) for early respondents $(\mathrm{M}=4.013, \mathrm{SD}=0.7515)$ and late respondents $(\mathrm{M}=4.155, \mathrm{SD}=0.7732)$. However, for the rest of 27 indicators, the t-test result does not find significant difference between the two respondent groups. Consequently, nonresponse bias does not appear to be a major problem for the whole research while caution should be exercised in applying the findings.

\subsection{Sample size and data screening}

In our structural model, the maximum number of arrows pointing at a construct is five. In order to detect minimum $\mathrm{R}^{2}$ value of 0.10 in any of the constructs for a significant level of 
$1 \%$, the minimum sample size required is 205 based on [54]. Since we have 740 usable responses, the minimum sample size requirement is thus met.

Data screening is performed using SPSS21. Missing data for an observation exceeding $10 \%$ are removed, and other missing values are replaced by using the mean value replacement. Although PLS-SEM does not require data to be normally distributed [54], normality is checked to ensure that the data are not too far away from normal distribution to affect the assessment of the parameters' significances. Of all 29 indicators, 26 of them are normally distributed, while three (FC1, KPI1, OPEN1) are not. This deviation from normality is not considered a major issue in this study.

\subsection{Exploratory factor analysis on BA applications}

BA includes different techniques. In order to explore the dimensions of BA and classify various types of BA into meaningful categories, we conduct an exploratory factor analysis (EFA) using a principal component analysis with Varimax rotation (SPSS21). Consequently, three factors are identified from 13 BA techniques with $62.72 \%$ of total variance explained. The first factor includes four BA techniques: statistical analysis, forecasting, query and analysis, and business reporting/KPIs. Since these statistical approaches are commonly used by organisations, thus we broadly name them as commonly used BA (CBA). The second factor includes six BA techniques: model management, optimisation, predictive modelling, simulation, interactive data visualisation, and data and text mining. We name them as modelbased BA (MBA) since modelling is the uniform essence of all these techniques. The third factor includes web analytics, social media analytics, and text-audio-video analytics. We name them as web-oriented BA (WBA) as they are used for analysing clickstream data and information collected mainly on the web. We are aware that while this classification provides a useful broad categorisation to facilitate communication, it needs to be further improved. For 
example, MBA includes data-and text mining that could be part of WBA and some organisations use web analytics more commonly. Detailed BA applications for each group are shown in Table 4.

Table 4. EFA analysis of BA applications

\begin{tabular}{|l|c|c|c|c|}
\hline \multirow{2}{*}{ BA Tools/Techniques } & \multicolumn{2}{|c|}{ Components and Factor Loadings } & \multirow{2}{*}{ Communalities } \\
\cline { 2 - 4 } & CBA & MBA & WBA & 0.564 \\
Statistical analysis & $\mathbf{0 . 6 8}$ & & & 0.609 \\
Forecasting & $\mathbf{0 . 7 5}$ & & & 0.404 \\
Query and analysis & $\mathbf{0 . 5 2}$ & 0.33 & & 0.637 \\
Business reporting / KPIs & $\mathbf{0 . 7 8}$ & & & 0.717 \\
Model management & & $\mathbf{0 . 7 8}$ & & 0.561 \\
Optimisation & 0.35 & $\mathbf{0 . 6 5}$ & & 0.641 \\
Predictive modelling & 0.45 & $\mathbf{0 . 6 5}$ & & 0.654 \\
Simulation & & $\mathbf{0 . 7 5}$ & & 0.619 \\
Interactive data visualisation & & $\mathbf{0 . 6 6}$ & 0.43 & 0.408 \\
Data and text mining & 0.36 & $\mathbf{0 . 3 9}$ & 0.36 & 0.709 \\
Web analytics & & & $\mathbf{0 . 8 0}$ & 0.769 \\
Social media analytics & & & $\mathbf{0 . 8 5}$ & 0.642 \\
Text-audio-video analytics & & 0.48 & $\mathbf{0 . 6 1}$ & \\
\hline
\end{tabular}

The EFA results are assessed based on the threshold values suggested by [62]. The associated KMO with the EFA is 0.89 , which is acceptable; Bartlett's Test is significant at $p<0.000$, and all communalities are above 0.4 , suggesting the appropriateness of the data. Cronbach's alpha is 0.88 , suggesting reliability. All factor loadings are above 0.30 with a sample of 740, suggesting convergent validity. In addition, the three factors identified namely CBA, MBA, and WBA make sense because variables similar in nature loaded together on the same factor, suggesting face validity. However, discriminant validity is not entirely satisfactory since three variables including query and analysis, text-audio-video analytics, and data and text mining have cross-loadings that are not different by more than 0.2 . Yet, these three variables are retained since they provide useful information about BA and this is an exploratory research in nature.

Apart from developing a BA classification, this EFA analysis has also confirmed our previous discussion in Section 3.1 that BA should be defined as a multidimensional construct. 
Therefore, BA as a higher-order formative construct is finalised and defined by three lowerorder reflective constructs, namely, CBA, MBA, and WBA.

\subsection{Evaluation of the reflective measurement indicators}

Our PLS-SEM model includes both formative and reflective constructs (only lower-order components). Following the recommendations made by [54], the reflective measurement model is evaluated by considering the internal consistency (composite reliability), indicator reliability, convergent validity and discriminant validity.

Composite reliability (CR) scores summarised in Table 5 indicate that results based on these constructs are consistent since all constructs meet the recommended threshold value for acceptable reliability, that is, both CR and Cronbach's $\alpha$ should be large than 0.70 .

Table 5. Convergent validity and internal consistency reliability

\begin{tabular}{|c|c|c|c|c|c|c|}
\hline Construct & Indicator & Loading & $\begin{array}{l}\text { Indicator } \\
\text { reliability }\end{array}$ & $\begin{array}{l}\text { Composite } \\
\text { reliability }\end{array}$ & $\begin{array}{l}\text { Cronbach's } \\
\text { alpha }\end{array}$ & AVE \\
\hline \multirow{6}{*}{ MBA } & DTM1 & 0.64 & 0.41 & \multirow{6}{*}{0.89} & \multirow{6}{*}{0.84} & \multirow{6}{*}{0.56} \\
\hline & IDV1 & 0.70 & 0.49 & & & \\
\hline & MM1 & 0.83 & 0.69 & & & \\
\hline & OPT1 & 0.75 & 0.56 & & & \\
\hline & PM1 & 0.79 & 0.62 & & & \\
\hline & SM1 & 0.78 & 0.61 & & & \\
\hline \multirow{4}{*}{ CBA } & FC1 & 0.74 & 0.55 & \multirow{4}{*}{0.83} & \multirow{4}{*}{0.73} & \multirow{4}{*}{0.55} \\
\hline & KPI1 & 0.75 & 0.56 & & & \\
\hline & QA1 & 0.71 & 0.50 & & & \\
\hline & SA1 & 0.78 & 0.61 & & & \\
\hline \multirow{3}{*}{ WBA } & SMA1 & 0.85 & 0.72 & \multirow{3}{*}{0.86} & \multirow{3}{*}{0.76} & \multirow{3}{*}{0.68} \\
\hline & TAVA1 & 0.80 & 0.64 & & & \\
\hline & WA1 & 0.82 & 0.67 & & & \\
\hline
\end{tabular}

Indicator reliability is first assessed by observing the factor loadings and each indicator's variance, the former should be large than 0.70 and the latter should be no less than 0.50. All factor loadings are above 0.7 except that DTM1's loading is close to 0.7 and IDV1's loading is 0.7; and all variances are above 0.5 except that the variances of IDV1 and DTM1 are below 0.5 . Therefore, indicator reliability is not entirely satisfactory but acceptable. 
Convergent validity is also satisfactory since the average variance extracted (AVE) value for each construct in Table 5 is no less than the recommended threshold value of 0.50 .

Discriminant validity is satisfactory based on two tests. The first test is to analyse Fornell-Larcker criterion [50] to evaluate if the square root of AVE value for each construct is greater than the correlation of the construct with any other construct, which is true based on the comparison summarised in Table 6.

Table 6. Inter-construct correlations

\begin{tabular}{|l|c|c|c|}
\hline & WBA & MBA & CBA \\
\hline WBA & $\mathbf{0 . 8 1}$ & & \\
\hline MBA & 0.58 & $\mathbf{0 . 7 4}$ & \\
\hline CBA & 0.40 & 0.63 & $\mathbf{0 . 7 4}$ \\
\hline
\end{tabular}

Square root of $A V E$ on the diagonal

The second test is to observe if each reflective indicator loads highest on the construct it is associated with, which is also true (Table 7), thus demonstrating discriminant validity is satisfactory.

Table 7. Cross-loading analysis

\begin{tabular}{|l|c|c|c|}
\hline & WBA & MBA & CBA \\
\hline SMA1 & $\mathbf{0 . 8 5}$ & 0.40 & 0.32 \\
\hline TAVA1 & $\mathbf{0 . 8 0}$ & 0.52 & 0.32 \\
\hline WA1 & $\mathbf{0 . 8 2}$ & 0.43 & 0.33 \\
\hline DTM1 & 0.44 & $\mathbf{0 . 6 4}$ & 0.43 \\
\hline IDV1 & 0.52 & $\mathbf{0 . 7 0}$ & 0.36 \\
\hline MM1 & 0.48 & $\mathbf{0 . 8 3}$ & 0.46 \\
\hline OPT1 & 0.33 & $\mathbf{0 . 7 5}$ & 0.48 \\
\hline PM1 & 0.37 & $\mathbf{0 . 7 9}$ & 0.57 \\
\hline SM1 & 0.32 & $\mathbf{0 . 7 8}$ & 0.45 \\
\hline FC1 & 0.24 & 0.44 & $\mathbf{0 . 7 4}$ \\
\hline SA1 & 0.35 & 0.53 & $\mathbf{0 . 7 8}$ \\
\hline KPI1 & 0.27 & 0.38 & $\mathbf{0 . 7 5}$ \\
\hline QA1 & 0.30 & 0.49 & $\mathbf{0 . 7 1}$ \\
\hline
\end{tabular}

\subsection{Assessment of formative measurement indicators}

The formative measurement model is evaluated in terms of collinearity, the indicator 
weights, significance of weights, and the indicator loadings [54]. To assess the level of collinearity, the variance inflation of factor (VIF) values of all formative constructs are evaluated (Table 8). The threshold value suggested for VIF is 3.3 by [56] and 5 by [54]; thus, there are no collinearity issues.

Table 8. Collinearity assessment

\begin{tabular}{|l|l|l|l|l|l|}
\hline \multicolumn{2}{|c|}{ BA } & \multicolumn{2}{c|}{ IPC } & \multicolumn{2}{c|}{ DDE } \\
\hline Indicators & VIF & Indicators & VIF & Indicators & VIF \\
\hline WBA & 1.462 & CD1 & 3.123 & STRA1 & 2.920 \\
MBA & 2.022 & ID1 & 3.611 & POL1 & 2.347 \\
CBA & 1.653 & AD1 & 2.306 & STRU1 & 2.492 \\
\cline { 1 - 1 } DDM & UD1 & 2.240 & PRO1 & 3.203 \\
\cline { 1 - 4 } Indicators & VIF & \multicolumn{2}{c}{ DME } & PERF1 & 2.168 \\
\hline S/P1 & 1.711 & Indicators & VIF & & \\
\cline { 3 - 4 } DM1 & 1.738 & CHA1 & 2.924 & & \\
DATA1 & 1.055 & RTD1 & 3.226 & & \\
OPEN1 & 1.160 & CUS1 & 2.487 & & \\
\cline { 1 - 3 }
\end{tabular}

Based on the bootstrapping process (5,000 samples), all formative indictors' outer loadings, outer weights and the associated significance testing $p$-values are assessed and summarised in Table 9. Except for AD1 and CUS1, all other indicators' outer weights are significant. When a formative indicator's outer weight is not significant, [54] suggests that it should be kept if its outer loading is above 0.5. As AD1 and CUS1's outer loadings are above 0.5 , they are retained, demonstrating each indicator's absolute contribution to the associated formative construct.

\subsection{Hypothesis testing}

SmartPLS 3 is used for testing the hypotheses and the results are presented in Figure 2.

Following [54], the structural model is assessed in terms of collinearity and the significance and relevance of the structural model relationships. To assess collinearity issues, four sets of predictor constructs are evaluated in SPSS 21 based on the latent variable scores from SmartPLS 3. The VIF values are summarised in Table 10 and there are no collinearity issues. 
Table 9. Outer weights \& significance testing results

\begin{tabular}{|c|c|c|c|c|}
\hline $\begin{array}{l}\text { Formative } \\
\text { Constructs }\end{array}$ & $\begin{array}{l}\text { Formative } \\
\text { Indicators }\end{array}$ & $\begin{array}{l}\text { Outer } \\
\text { Weights }\end{array}$ & $p$-values & $\begin{array}{l}\text { Outer } \\
\text { Loadings }\end{array}$ \\
\hline \multirow{3}{*}{ BA } & WBA & 0.17 & $0.0000^{* * * *}$ & 0.66 \\
\hline & MBA & 0.56 & $0.0000^{* * *}$ & 0.93 \\
\hline & CBA & 0.44 & $0.0000^{* * *}$ & 0.85 \\
\hline \multirow{5}{*}{ DDE } & PERF1 & 0.27 & $0.0000^{* * * *}$ & 0.83 \\
\hline & POL1 & 0.16 & $0.0139^{*}$ & 0.81 \\
\hline & PRO1 & 0.31 & $0.0000^{* * *}$ & 0.90 \\
\hline & STRA1 & 0.15 & $0.0305^{*}$ & 0.86 \\
\hline & STRU1 & 0.28 & $0.0000^{* * *}$ & 0.85 \\
\hline \multirow{4}{*}{ IPC } & AD1 & 0.05 & $0.5783^{\mathrm{ns}}$ & 0.77 \\
\hline & CD1 & 0.24 & $0.0094^{* *}$ & 0.87 \\
\hline & ID1 & 0.38 & $0.0001^{* * *}$ & 0.92 \\
\hline & UD1 & 0.45 & $0.0000^{* * *}$ & 0.90 \\
\hline \multirow{4}{*}{ DDM } & DATA1 & 0.49 & $0.0000^{* * * *}$ & 0.66 \\
\hline & DM1 & 0.32 & $0.0000^{* * * *}$ & 0.74 \\
\hline & OPEN1 & 0.28 & $0.0000^{* * *}$ & 0.57 \\
\hline & $\mathrm{S} / \mathrm{P} 1$ & 0.38 & $0.0000^{* * * *}$ & 0.74 \\
\hline \multirow{3}{*}{ DME } & CHA1 & 0.28 & $0.0157^{*}$ & 0.89 \\
\hline & CUS1 & 0.18 & $0.1426^{\mathrm{ns}}$ & 0.83 \\
\hline & RTD & 0.62 & $0.0000^{* * * *}$ & 0.96 \\
\hline
\end{tabular}

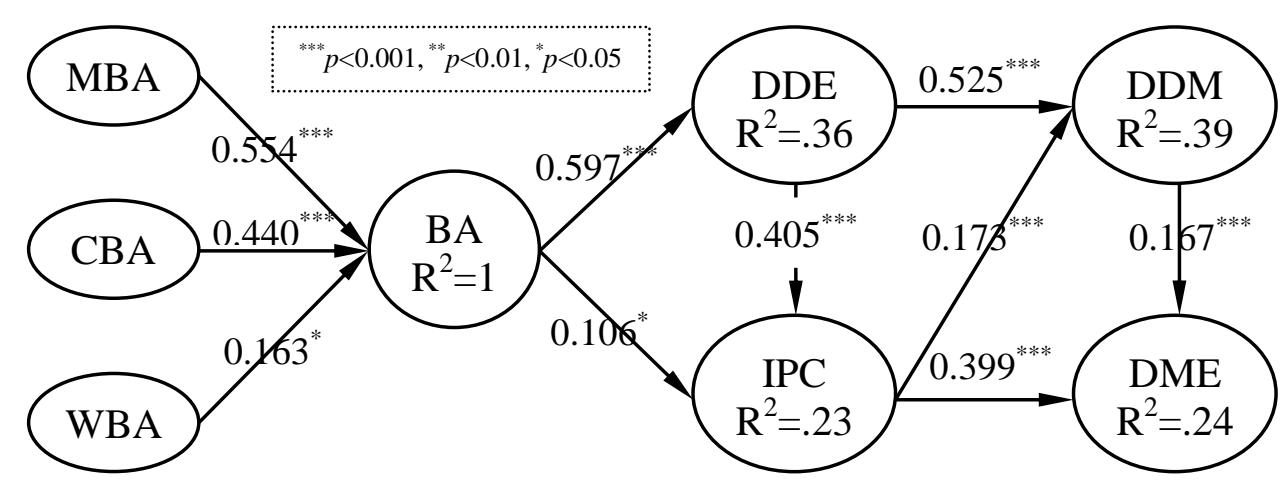

Figure 2. Final research model and path analysis results

Table 10. Collinearity assessment in the formative measurement model

\begin{tabular}{|l|l|l|l|l|l|l|l|}
\hline \multicolumn{2}{|c|}{$1^{\text {st }}$ set } & \multicolumn{2}{c|}{$2^{\text {nd }}$ set } & \multicolumn{2}{c|}{$3^{\text {rd }}$ set } & \multicolumn{2}{c|}{$4^{\text {th }}$ set } \\
\hline Construct & VIF & Construct & VIF & Construct & VIF & Construct & VIF \\
\hline MBA & 2.14 & BA & 1.56 & DDE & 1.26 & DDM & 1.20 \\
CBA & 1.64 & DDE & 1.56 & IPC & 1.26 & IPC & 1.20 \\
\cline { 3 - 8 } & 1.65 & \multicolumn{5}{ll}{} \\
\cline { 3 - 7 }
\end{tabular}


The significance and relevance of the path coefficients are shown in Figure 2. BA is significantly related to both data-driven environment (DDE) and information processing capabilities (IPC). Data-driven environment is strongly related to information processing capabilities, which in turn are significantly related to data-driven decision-making (DDM) and decision-making effectiveness (DME). Data-driven decision-making is significantly contributing to decision-making effectiveness.

From Table 11, information processing capabilities have the strongest total effect on decision-making effectiveness, followed by data-driven environment, BA, and data-driven decision-making. A data-driven environment has the strongest total effect on information processing capabilities, followed by BA.

Table 11. Total effect

\begin{tabular}{|l|l|l|l|c|c|}
\hline \multicolumn{3}{|c|}{ Total effect on DME } & \multicolumn{2}{c|}{ Total effect on IPC } \\
\hline IPC & DDE & BA & DDM & DDE & BA \\
\hline 0.43 & 0.26 & 0.20 & 0.17 & 0.41 & 0.35 \\
\hline
\end{tabular}

The predictive power of the model can be assessed by observing the amount of variance attributed to the latent variables (i.e., $\mathrm{R}^{2}$ ) and the value of the predictive relevance $\mathrm{Q}^{2}$, summarised in Table 12.

Table 12. Results of $R^{2}$ and $Q^{2}$ values

\begin{tabular}{|l|l|l|l|l|l|}
\hline & IPC & DDE & BA & DDM & DME \\
\hline $\mathrm{R}^{2}$ Value & 0.23 & 0.36 & 1.0 & 0.39 & 0.24 \\
\hline $\mathrm{Q}^{2}$ Value & 0.17 & 0.26 & 0.42 & 0.18 & 0.19 \\
\hline
\end{tabular}

All $\mathrm{Q}^{2}$ in Table 12 are above zero, providing support for the model's predictive relevance regarding the latent variables [54]. The model's predictive power is reflected by the variables' $\mathrm{R}^{2}$ values. When PLS-SEM is used in IT studies, the effect size suggested for $\mathrm{R}^{2}$ is small $=0.1$, medium $=0.25$, and large $=0.36$ [51]. In line with this, the effect sizes of DDE and DDM can be classified as large; the effect sizes of DME and IPC are close to medium. The 
overall model's explanatory power being $24 \%$ suggests that there are other influencing factors beyond the scope of our research model to affect the organisation's decision-making effectiveness. Such factors may include for example characteristics of top management team, organisational structure, and business environment [63].

Table 13 summarise the results of hypothesis testing with the standardised path coefficients and $p$-values where appropriate.

Table 13. Summary results of hypotheses testing

\begin{tabular}{|c|c|c|c|c|}
\hline & Hypothesised path & Stand. path coefficient & $p$-values & Hypothesis test \\
\hline H1 & $\mathrm{BA}->\mathrm{IPC}$ & 0.106 & $0.0111^{*}$ & Supported \\
\hline $\mathbf{H 2}$ & BA -> DDE->IPC & & & Supported \\
\hline $\mathbf{H 3}$ & IPC -> DDM & 0.173 & $0.0000^{* * * *}$ & Supported \\
\hline H4 & IPC -> DME & 0.399 & $0.0000^{* * * *}$ & Supported \\
\hline H5 & DDE -> DDM & 0.525 & $0.0000^{* * * *}$ & Supported \\
\hline H6 & DDM -> DME & 0.167 & $0.0002^{* * * *}$ & Supported \\
\hline H7 & \multicolumn{3}{|c|}{ Firm size moderates the proposed path } & Rejected \\
\hline H8 & \multicolumn{3}{|c|}{ Industry type moderates the proposed path } & Weakly supported \\
\hline
\end{tabular}

H1 suggests that BA has a positive and direct effect on information processing capabilities (IPC), which is supported as BA's effect on IPC is $0.106(p<0.05)$. H2 assumes that BA has an indirect effect on IPC through the mediation of a data-driven environment (DDE). To validate $\mathrm{H} 2$, the mediating role of data-driven environment on the relationship between BA and information processing capabilities was analysed, following the steps suggested by [64] but based on bootstrapping [54]. The relative size of the mediating effect is decided by calculating the variance accounted for (VAF) [65]. The result of the analysis summarised in Table 14 suggests that data-driven environment partially but strongly mediates the effect of BA on information processing capabilities; thus, $\mathrm{H} 2$ is supported.

H3 suggests that information processing capabilities have a positive effect on datadriven decision-making (DDM). As shown in Table 13, the effect of information processing capabilities on data-driven decision-making is $0.173(p<0.001)$; thus H3 is supported. H4 
Table 14. The mediating role of a data-driven environment

\begin{tabular}{|l|l|l|l|l|l|}
\hline Hypothesis & $\begin{array}{l}\text { Direct effect } \\
\text { without mediation }\end{array}$ & $\begin{array}{l}\text { Direct effect } \\
\text { with mediation }\end{array}$ & $\begin{array}{l}\text { Indirect } \\
\text { effect }\end{array}$ & VAF & $\begin{array}{l}\text { Mediation } \\
\text { type observed }\end{array}$ \\
\hline H2 & $0.347^{* * * *}$ & $0.106^{*}$ & $0.242^{* * *}$ & 0.695 & Partial \\
\hline
\end{tabular}

posits that information processing capabilities have a direct and positive effect on decisionmaking effectiveness (DME), which is supported as the effect of information processing capabilities on decision-making effectiveness is 0.399 at $(p<0.001)$. H5 suggests that datadriven environment has a direct and positive effect on data-driven decision-making, which in turn has a positive effect on decision-making effectiveness (H6). We find that the direct effect of data-driven environment on data-driven decision-making is $0.525(p<0.001)$ and the effect of data-driven decision-making on decision-making effectiveness is $0.167(p<0.001)$; thus, both H5 and H6 are supported.

$\mathrm{H} 7$ and $\mathrm{H} 8$ propose that firm size and industry type moderate the paths from BA to DME respectively. To understand whether firm size or industry type moderates the paths from BA to decision-making effectiveness, a PLS-SEM multi-group analysis (PLS-MGA) is conducted. When engaging in PLS-MGA, the number of observations in each group also needs to meet the minimum sample size requirement. In order to detect a minimum $\mathrm{R}^{2}$ value of 0.25 in any of the constructs for a significant level of $1 \%$, the minimum sample size required is 98 since the maximum number of arrows pointing at a construct is five in this research [54]. Thus, to test the moderating effect of firm size, we compare medium $(n=524)$ and large $(n=216)$ companies; to test the moderating effect of industry type, we compare manufacturing $(n=232)$ and professional services $(n=108)$ industries. We are unable to compare others because the sample size for each of the other industries is below 98 . The comparison between the hypothesised paths of medium $(n=524)$ and large $(n=216)$ companies indicates that company size has no moderating effect on the paths from BA to decision- 
making effectiveness because none of the $p$-values associated with the comparison is significant; thus, $\mathrm{H} 7$ is rejected

Similarly, in order to assess the moderating effect of industry type on the paths from BA to decision-making effectiveness, the hypothesised paths are compared between manufacturing $(n=232)$ and professional services $(n=108)$. The comparison identifies that two of the seven paths are significantly different: the comparison $p$-value associated with the DDM to DME path is $0.012<0.05$ while the comparison $p$-value associated with the IPC to DDM path is $0.044<0.05$. Thus, there is some statistical difference between the paths of professional and manufacturing; H9 is weakly supported.

\subsection{Testing the exogeneity of explanatory variables}

Before we proceed to interpret the findings, a potential problem with our research is the endogeneity of explanatory variables in the research model, which may introduce a serious bias [66] that makes inferences problematic [67]. To validate our research, a Hausman test is conducted to show the exogeneity of the explanatory variables and the absence of the correlation between the explanatory variables and the error terms. This test uses instrumental variable (IV), which must be (a) strongly correlated with the independent variable and (b) independent of the error terms [68]. Three IVs are identified. The first IV is identifying problems and opportunities, which could result in the use of BA but is unlikely to lead to a data-driven environment (DDE) directly as the latter refers to the organisational strategy, structure, and business processes that are specifically developed to support and enable BA applications. A correlation analysis confirms that this IV is related to BA ( $p<0.05,2$-tailed) but not to DDE; therefore, identifying problems and opportunities is used as an IV for BA. The second IV is routing gathering of opinions from clients that is part of BA application, which helps develop a data-driven environment based on contingency theory as we have discussed previously in section 2.2. Thus, the second IV is expected to relate to DDE but not 
to data-driven decision-making (DDM) directly since the latter is defined by a number of other factors, though it may use the insights gained from routing gathering of opinions from clients as part of decision input. A correlation analysis confirms that routing gathering of opinions from clients is related to DDE ( $p<0.05,2$-tailed) but not to DDM. Thus, routing gathering of opinions from clients is used as an IV for DDE. The third IV identified is the use of predictive analytics that is seen to provide useful input to support DDM but not likely to directly affect decision-making effectiveness (DME) since the latter is the combined result of data-driven decision-making and other organisational factors. A correlation analysis indicates that the use of predictive analytics is related to DDM $(p<0.05,2$-tailed $)$ but not to DME. Therefore, we use predictive analytics as IV for DDM. However, we are unable to find suitable IV for information processing capabilities (IPC). Using the three IVs, we attempt to conduct the Hausman test for each of the following four paths: BA $\rightarrow$ DDE, BA + DDE $\rightarrow$ IPC, $\mathrm{DDE}+\mathrm{IPC} \rightarrow \mathrm{DDM}$, and DDM+IPC $\rightarrow$ DME. The test result listed in Table 15 indicates that the explanatory variables in our model are not significantly endogenous, except that we are unable to test the exogeneity of IPC. Thus, the PLS estimation is seen to be acceptable.

Table 15. Hausman test for endogeneity

\begin{tabular}{|l|l|c|c|c|}
\hline Path & Instrumental variable & Hausman statistics & Df & $p$-value \\
\hline $\mathrm{BA} \rightarrow \mathrm{DDE}$ & $\begin{array}{l}\text { identifying problems and } \\
\text { opportunities (for BA) }\end{array}$ & 2.8939 & 2 & 0.2353 \\
\hline $\mathrm{BA}+\mathrm{DDE} \rightarrow \mathrm{IPC}$ & $\begin{array}{l}\text { identifying problems and } \\
\text { opportunities (for BA) \& } \\
\text { routing gathering of opinions } \\
\text { from clients (for DDE) }\end{array}$ & 0.3243 & 3 & 0.9554 \\
\hline $\mathrm{DDE}+\mathrm{IPC} \rightarrow \mathrm{DDM}$ & $\begin{array}{l}\text { routing gathering of opinions } \\
\text { from clients (for DDE) }\end{array}$ & 0.3732 & 3 & 0.9457 \\
\hline $\mathrm{DDM}+\mathrm{IPC} \rightarrow \mathrm{DME}$ & $\begin{array}{l}\text { the use of predictive analytics } \\
\text { (for DDM) }\end{array}$ & 2.2683 & 3 & 0.5186 \\
\hline
\end{tabular}

\section{DISCUSSION AND CONCLUSION}


The motivation of this study is to develop an understanding of the mechanisms through which BA improves decision-making effectiveness. While BA has recently re-emerged as an important area of study [3, 4]; little is known about BA's impact on organisational decisionmaking as little academic research has been conducted to date $[5,13]$.

This study has based on prior BA literature, the information processing view and contingency theory to develop a path model to conceptualise and examine relevant concepts pertaining to BA and its impact on decision-making effectiveness. In the process of enhancing understanding of these concepts and their relationships, we believe our research offers original insights into how BA improves decision-making effectiveness.

First, we contribute to the literature on BA by developing an understanding of the mechanisms through which BA improves decision-making effectiveness. Although the importance of developing a data-driven environment and the potential of using BA to create business value have been indicated by prior studies $[8,9,20]$, there is little conceptual understanding and empirical evidences to validate these assertions. This research advances our knowledge by developing a conceptual understanding underpinned by relevant theories and providing empirical evidence. By conceptualising the links between BA and decisionmaking effectiveness, our research directs attention to the complex interdependences between different organisational factors and the processes underlying BA applications. We have also provided empirical evidence to support the conceptualisation. The research findings show that BA has a positive effect on information processing capabilities directly and indirectly through the mediation of a data-driven environment. Then information processing capabilities will have a positive effect on data-driven decision-making, which positively improve decision-making effectiveness. On the one hand, this finding confirms the suggestions made in prior studies $[8,9,20]$ that BA positively enhances information processing capabilities. On the other hand and more importantly, the findings show that, in addition to a direct effect, BA 
positively influences data-driven environment. By explicating such mechanisms, our research suggests that applying BA requires an organisation to have a data-driven environment simultaneously to support and enable BA activities; otherwise, BA applications are likely to be unproductive. We hope that our conceptualisation of the relationships between BA and other organisational factors will inspire others to conduct more research so that a deeper understanding of the domain can be developed.

Second, we add to the on-going debate surrounding the proposition that IT can be an important determinant of organisational factors underpinned by contingency theory [e.g., 32, 34]. Our empirical evidence suggests that facing the challenges of big data, increasing competition, and technological advancement, BA applications and their benefits will help organisations to realise that it is advantageous to develop appropriate strategy, structure and processes to guide and enable BA activities. Thus, an important implication of our study is the need to conduct more research on how BA helps develop a data-driven environment in an organisation thereby to better support its decision-making.

Third, we contribute to the information processing view by providing empirical evidence to support the key idea that an organisation needs to design its structure [17] and business processes [18] to improving its information processing capabilities thereby to improve its decision-making [16]. Our research through the concept of a data-driven environment and empirical evidence suggests that when an organisation has developed specific strategy, policy, structure, and processes to enable BA activities, its information processing capabilities can be enhanced to improve its decision-making. Therefore, in addition to the idea that $\mathrm{BA}$ is an important factor for the development of a data-driven environment underpinned by contingency theory, our research drawing on the information processing view further supports that creating a data-driven environment in an organisation will help improve the organisation's information processing capabilities and ultimately its 
decision-making effectiveness. Consequently, this research underpinned by both contingency theory and the information processing view enhances our understanding of the mechanisms of BA's impact on decision-making.

Fourth, we provide useful insights into whether company size and industry type moderate the paths from BA to decision-making effectiveness. Our findings indicate that large and medium companies use BA similarly to support decision-making. This is not actually in conflict with $[40,41]$ since we have not included small business that are expected to behave differently regarding IT use, compared with large companies. However, our findings indicate that there are some differences across industries regarding BA applications. While our methodology does not allow us to provide an in-depth explanation of this finding, it provides empirical evidence in the context of BA to weakly support the moderating effect of industry type $[47,48]$. This tentative result calls for more research to develop a deeper understanding of how different industries use BA.

Our research findings also provide important implications for BA practitioners. The findings suggest that in an organisation BA is an important determinant of a data-driven environment, which is the necessary condition for effective BA applications and decisionmaking. Thus, BA must be implemented in tandem with developing a data-driven environment to realise its potential. A data-driven environment would enhance BA's impact on the organisation's information processing capabilities, which in turn, influence data-driven decision-making and decision-making effectiveness. Therefore, companies should focus on developing information processing capabilities with BA applications in a data-driven organisational environment.

The study has several limitations. First, although we have followed the four decision rules [56] to develop formative constructs to avoid misspecifications, we are unable to assess the convergent validity of the formative constructs to evaluate whether the entire domain of 
each formative construct has been covered by the selected indictors because the research design does not include additional reflective items or "shadow" reflective constructs that capture the essences of all five formative constructs. Second, our sample does not include small enterprises with less than 50 employees. Thus, our findings are not applicable to small enterprises. Third, we have used perceived measures to understand the key variables in this research while quantitative measures based on specific decisions may complement the perceived measures. Finally, we have not tested the exogeneity of information processing capabilities since we are unable to find suitable instrumental variable for this constructs.

Despite these limitations, however, we believe our study offers opportunities for future research. First, the understanding of BA and its impact could be further advanced by conducting more context-specific (such as a particular industry) investigations, thereby help companies to make better decisions about their investment. A second area for future research is to understand the status of BA applications and its impact on decision-making in small businesses. Third, factors such as top management team, organisational structure, and business environment may have a significant effect on shaping the outcomes of strategic decisions and thus should be examined in future BA research. Finally, in order for researchers to have more confidence in drawing conclusions from research, future empirical IT research should begin to address the issue of endogeneity as it could lead to biased and inconsistent estimators thereby to limit the validity of research models.

\section{REFERENCES}

[1]T. H. Davenport and J. G. Harris, Competing on Analytics: The New Science of Winning. Boston, MA: Harvard Business School Review Press, 2007.

[2]T. H. Davenport, "Analytics 3.0," Harvard Business Review, vol. 91, pp. 64-72, 2013.

[3]H. Chen, R. H. L. Chiang, and V. C. Storey, "Business intelligence and analytics: from big data to big impact," MIS Quarterly, vol. 36, pp. 1165-1188, 2012.

[4]H. J. Watson, "Tutorial: Big Data Analytics: Concepts, Technologies, and Applications," Communications of the Association for Information Systems, vol. 34, pp. 1247-1268, 2014.

[5]C. Holsapple, A. Lee-Post, and R. Pakath, "A unified foundation for business analytics," Decision Support Systems, 2014.

[6]T. H. Davenport, "Competing on analytics," Harvard Business Review, vol. 84, pp. 98-107, 2006. 
[7]T. H. Davenport, J. G. Harris, D. W. De Long, and A. L. Jacobson, "Data to Knowledge to Results: Building an analytic capability," California Management Review, vol. 43, pp. 117-138, 2001.

[8]D. Kiron, P. K. Prentice, and R. B. Ferguson, "Innovating With Analytics. (cover story)," MIT Sloan Management Review, vol. 54, pp. 47-52, 2012.

[9]S. Lavalle, E. Lesser, R. Shockley, M. S. Hopkins, and N. Kruschwitz, "Special Report: Analytics and the New Path to Value," MIT Sloan Management Review, vol. 52, pp. 22-32, 2011.

[10] D. Kiron, P. K. Prentice, and R. B. Ferguson, "Raising the Bar With Analytics," MIT Sloan Management Review, vol. 55, pp. 29-33, 2014.

[11] D. Barton and D. Court, "Making Advanced Analytics Work For You," Harvard Business Review, vol. 90, pp. 78-83, 2012.

[12] K. Gillon, S. Aral, L. Ching-Yung, S. Mithas, and M. Zozulia, "Business Analytics: Radical Shift or Incremental Change?," Communications of the Association for Information Systems, vol. 34, pp. 287-296, 2014.

[13] G. George, M. R. Haas, and A. Pentland, "Big data and management," Academy of Management Journal, vol. 57, pp. 321-326, 2014.

[14] D. A. Marchand and J. Peppard, "Why IT Fumbles Analytics," Harvard Business Review, vol. 91, pp. 104-112, 2013.

[15] K. M. Eisenhardt and M. J. Zbaracki, "Strategic Decision Making," Strategic Management Journal, vol. 13, pp. 17-37, 1992.

[16] J. R. Galbraith, "Organization design: an information processing view," Interfaces, vol. 4, pp. 2836, 1974.

[17] M. L. Tushman and D. A. Nadler, "Information Processing as an Integrating Concept in Organizational Design," Academy of Management Review, vol. 3, pp. 613-624, 1978.

[18] G. Premkumar, K. Ramamurthy, and C. S. Saunders, "Information Processing View of Organizations: An Exploratory Examination of Fit in the Context of Interorganizational Relationships," Journal of Management Information Systems, vol. 22, pp. 257-294, 2005.

[19] E. T. Wang, J. C. F. Tai, and V. Grover, "Examining the relational benefits of improved interfirm information processing capability in buyer-supplier dyads," MIS Quarterly, vol. 37, pp. 149-173, 2013.

[20] D. Kiron and R. Shockley, "Creating Business Value Analytics," MIT Sloan Management Review, vol. 53, pp. 57-63, 2011.

[21] S. Lavalle, E. Lesser, R. Shockley, M. S. Hopkins, and N. Kruschwitz, "Big Data, Analytics and the Path from Insights to Value," MIT Sloan Management Review, vol. 52, pp. 21-31, 2011.

[22] F. Acito and V. Khatri, "Business analytics: Why now and what next?," Business Horizons (2014), , vol. 57, pp. 565-570, 2014.

[23] N. Rajagopalan, A. M. A. Rasheed, and D. K. Datta, "Strategic Decision Processes: Critical Review and Future Directions," Journal of Management, vol. 19, p. 349, 1993.

[24] D. A. Nadler and M. L. Tushman, "A Model for Diagnosing Organizational Behavior," Organizational Dynamics, vol. 9, pp. 35-51, 1980.

[25] H. L. Tosi and J. W. Slocum, "Contingency Theory: Some Suggested Directions," Journal of Management, vol. 10, pp. 9-26, 1984.

[26] Y. Chan, E. and B. H. Reich, "IT alignment: what have we learned?," Journal of Information Technology, vol. 22, pp. 297-315, 2007.

[27] P. Weill and M. H. Olson, "An Assessment of the Contingency Theory of Management Information Systems," Journal of Management Information Systems, vol. 6, pp. 59-85, 1989.

[28] J. Peppard and J. Ward, "Beyond strategic information systems: towards an IS capability," The Journal of Strategic Information Systems, vol. 13, pp. 167-194, 2004.

[29] J.-H. Lim, T. C. Stratopoulos, and T. S. Wirjanto, "Path Dependence of Dynamic Information Technology Capability: An Empirical Investigation," Journal of Management Information Systems, vol. 28, pp. 45-84, 2011.

[30] A. S. Bharadwaj, "A resource-based perspective on information technology capability and firm performance: an empirical investigation," MIS Quarterly, vol. 24, pp. 169-196, 2000.

[31] M. Jelinek, "Technology, Organizations, and Contingency," Academy of Management Review, vol. 2, pp. 17-26, 1977.

[32] J. Woodward, Management and Technology. London, UK.: H. M. S. O, 1958 
[33] J. D. Thompson and F. L. Bates, "Technology, Organization, and Administration," Administrative Science Quarterly, vol. 2, pp. 325-343, 1957.

[34] C. Perrow, "A Framework for the Comparative Analysis of Organizations," American Sociological Review, vol. 32, pp. 194-208, 1967.

[35] E. Sadler-Smith, "Cognitive Style and the Management of Small and Medium-Sized Enterprises," Organization Studies, vol. 25, pp. 155-181, 2004.

[36] S. B. Rodrigues and D. J. Hickson, "Success in decision making: different organizations, differing reasons for success," Journal of Management Studies, vol. 32, pp. 655-678, 1995.

[37] J. W. Dean Jr and M. P. Sharfman, "Does decision process matter? A study of strategic decisionmaking effectiveness," Academy of Management Journal, vol. 39, pp. 368-396, 1996.

[38] G. C. Mueller, M. A. Mone, and V. L. Barker Ill, "Formal Strategic Analyses and Organizational Performance: Decomposing the Rational Model," Organization Studies, vol. 28, pp. 853-883, 2007.

[39] I. Kun Shin, V. Grover, and J. T. C. Teng, "Do Large Firms Become Smaller by Using Information Technology?," Information Systems Research, vol. 24, pp. 470-491, 2013.

[40] W. H. Delone, "Firm Size and the Characteristics of Computer Use," Mis Quarterly, vol. 5, pp. 65-77, 1981.

[41] S. E. Harris and J. L. Katz, "Firm Size and the Information Technology Investment Intensity of Life Insurers," Mis Quarterly, vol. 15, pp. 333-352, 1991.

[42] J. Jayaram, S. L. Ahire, and P. Dreyfus, "Contingency relationships of firm size, TQM duration, unionization, and industry context on TQM implementation-A focus on total effects," Journal of Operations Management, vol. 28, pp. 345-356, 2010.

[43] P. M. Swamidass and S. Kotha, "Explaining manufacturing technology use, firm size and performance using a multidimensional view of technology," Journal of Operations Management, vol. 17, pp. 23-37, 1998.

[44] J. Lee, "Does Size Matter in Firm Performance? Evidence from US Public Firms," International Journal of the Economics of Business, vol. 16, pp. 189-203, 2009.

[45] L.-S. Lin, I.-C. Huang, P.-L. Du, and T.-F. Lin, "Human capital disclosure and organizational performanceThe moderating effects of knowledge intensity and organizational size," Management Decision, vol. 50, pp. 1790-1799, 2012.

[46] P. Neirotti and E. Paolucci, "Assessing the importance of industry in the adoption and assimilation of IT: Evidence from Italian enterprises," Information \& Management, vol. 48, pp. 249-259, 2011.

[47] S. B. Banerjee, E. S. Iyer, and R. K. Kashyap, "Corporate Environmentalism: Antecedents and Influence of Industry Type," Journal of Marketing, vol. 67, pp. 106-122, 2003.

[48] L. Y. M. Sin, A. C. B. Tse, O. H. M. Yau, R. P. M. Chow, and J. S. Y. Lee, "Market Orientation, Relationship Marketing Orientation, and Business Performance: The Moderating Effects of Economic Ideology and Industry Type," Journal of International Marketing, vol. 13, pp. 36-57, 2005.

[49] D. Gefen, E. E. Rigdon, and D. Straub, "An Update and Extension to SEM Guidelines for Administrative and Social Science Research," MIS Quarterly, vol. 35, pp. iii-A7, 2011.

[50] J. F. Hair, C. M. Ringle, and M. Sarstedt, "Partial Least Squares Structural Equation Modeling: Rigorous Applications, Better Results and Higher Acceptance," in Long Range Planning vol. 46, ed, 2013, pp. 1-12.

[51] M. Wetzels, G. Odekerken-Schröder, and C. van Oppen, "Using PLS path modeling for assessing hierarchical construct models: guidelines and empirical illustration," MIS Quarterly, vol. 33, pp. 177-195, 2009.

[52] W. W. Chin and P. R. Newsted, "Structural Equation Modeling analysis with Small Samples Using Partial Least Squares," in Statistical Strategies for Small Sample Research, R. Hoyle, Ed., ed Thousand Oaks, CA: Sage Publications, 1999, pp. 307-341.

[53] C. Fornell, "A Second Generation of Multivariate Analysis: Classification of Methods and Implications for Marketing Research," in Review of Marketing, M. Huston, Ed., ed Chicago: American Marketing Association, 1987.

[54] J. F. Hair, G. Hult, C. Ringle, and M. Sarstedt, A Primer on Partial Least Squares Structural Equation Modeling (PLS-SEM): Sage, 2014. 
[55] S. B. MacKenzie, P. M. Podsakoff, and N. P. Podsakoff, "Construct measurement and validation procedures in MIS and behavioral research: Integrating new and existing techniques," Mis Quarterly, vol. 35, pp. 293-A5, 2011.

[56] S. Petter, D. Straub, and A. Rai, "Specifying formative constructs in information systems research," Mis Quarterly, vol. 31, pp. 623-656, 2007.

[57] R. P. Bagozzi, Y. Youjae, and L. W. Phillips, "Assessing Construct Validity in Organizational Research," Administrative Science Quarterly, vol. 36, pp. 421-458, 1991.

[58] L. W. Phillips and R. P. Bagozzi, "On measuring organizational properties of distribution channels: methodological issues in the use of key informants," Research in Marketing, vol. 8, p. $313,1986$.

[59] N. K. Malhotra, A. Patil, and S. S. Kim, "BIAS Breakdown," Marketing Research, vol. 19, pp. 24-29, 2007.

[60] P. M. Podsakoff, S. B. MacKenzie, J.-Y. Lee, and N. P. Podsakoff, "Common method biases in behavioral research: A critical review of the literature and recommended remedies," Journal of Applied Psychology, vol. 88, pp. 879-903, 2003.

[61] J. S. Armstrong and T. S. Overton, "Estimating Nonresponse Bias in Mail Surveys," Journal of Marketing Research (JMR), vol. 14, pp. 396-402, 1977.

[62] J. F. Hair, W. C. Black, B. J. Babin, and R. E. Anderson, Multivariate Data Analysis, 7th ed.: Prentice Hall Higher Education, 2010.

[63] V. M. Papadakis, S. Lioukas, and D. Chambers, "Strategic decision-making processes: The role of management and context," Strategic Management Journal, vol. 19, p. 115, 1998.

[64] R. M. Baron and D. A. Kenny, "The Moderator-Mediator Variable Distinction in Social Psychological Research - Conceptual, Strategic, and Statistical Considerations," Journal of Personality and Social Psychology, vol. 5, pp. 1173-1182, 1986.

[65] P. E. Shrout and N. Bolger, "Mediation in experimental and nonexperimental studies: New procedures and recommendations," Psychological Methods, vol. 7, pp. 422-445, 2002.

[66] C. N. Mclntosh, J. R. Edwards, and J. Antonakis, "Reflections on Partial Least Squares Path Modeling," Organizational Research Methods, vol. 12, pp. 210-251, 2014.

[67] R. H. Chenhall and F. Moers, "The Issue of Endogeneity within Theory-Based, Quantitative Management Accounting Research," European Accounting Review, vol. 16, pp. 173-195, 2007.

[68] C. N. McIntosh, J. R. Edwards, and J. Antonakis, "Reflections on Partial Least Squares Path Modeling," Organizational Research Methods, vol. 17, pp. 210-251, 2014. 


\section{Managerial relevance statement}

Business analytics is being increasingly used to gain data-driven insights to support decisionmaking; however, extant literature indicates that many companies are still struggling to figure out how to use business analytics. Some of them are unsure how to proceed, while others are struggling to achieve a worthwhile return. The findings of this paper could provide valuable insights into how an organisation should use business analytics to improve its decision-making effectively. The findings of this paper show that a data-driven environment in an organisation is the core-facilitating factor for the application of business analytics and effective decision-making. In order to realise the potential of business analytics, an organisation must develop explicit organisational strategy and policy to guide analytic activities, and design its organisational structure and business processes to enable and facilitate data-driven decision-making. Our research findings suggest that a data-driven environment would enhance the impact of business analytics on the organisation's information processing capabilities, which in turn influence data-driven decision-making and decision-making effectiveness. Therefore, in order to realise the potentials of business analytics, companies should implement business analytics by developing an internal datadriven environment. 


\section{Biosketchs and photos}

Guangming Cao received both the BSc. degree in airplane design from the Airplane Department and the MSc. in management and system engineering from the Manufacturing Engineering Department, Northwestern Polytechnical University, Xi' an, China; and the Ph.D. degree in systems thinking and managing organisational change from the University of Luton, UK. He previously held lecturing positions at the Northwestern Polytechnical University in China and at the University of Ulster in UK. He is currently a Principal Lecturer at the University of Bedfordshire in UK. His research interests include business analytics, strategic IT alignment, IT business value, and systems thinking. His research has appeared in International Journal of Management Review, Supply Chain Management, Systemic Practice and Action Research, Strategic Change, and Systems Research and Behavioral Science.

Yanqing Duan, $\mathrm{PhD}$, is a Professor of Information Systems, Director of Business and Information Systems Research Centre (BISC) at the University of Bedfordshire, and a Visiting Professor of China Agricultural University. She received her BSc in Mechanical Engineering and MSs in Systems Engineering from China Agricultural University, and $\mathrm{PhD}$ from Aston Business School, Aston University in the UK. Her principal research interest is the use of the emerging Information and Communication Technologies (ICT) in organisations and their impact on

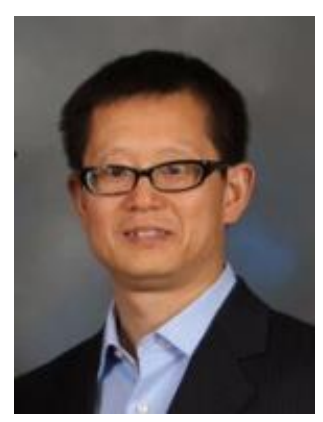
decision making, innovation, and knowledge management. This research focus is reflected in the context of ICT-based knowledge management and transfer, use of intelligent systems and Big Data Analytics in supporting decision making and innovation, and Small to Medium Sized Enterprises' (SMEs) adoption of digital business. She has co-ordinated many research projects funded by various funding bodies, mainly the European Commission. She published over 160 referred articles, including papers in European Journal of Information Systems, Information \& Management, European Journal of Marketing, Expert Systems with Applications, Information Technology \& People, etc.

Gendao Li received the B.S. degree in information management and systems, in 2004, and the Ph.D. degree in management science and engineering from Chongqing University, China.

He is currently a Senior Research Fellow at the University of Bedfordshire Business School. His current research interests include how to use big data analytics to improve company performance, including understanding the impact path from big data analytics to business performance, understanding consumer purchase behavior and optimizing pricing decisions. His work has been published in journals like European

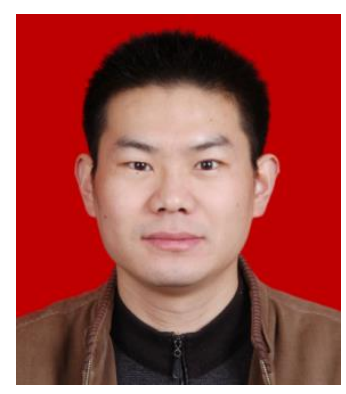
Journal of Operational Research, International Journal of Production Economics, Journal of the Operational Research Society, International Journal of Production Research. 